\title{
Differentiated rhabdomyomatous tumors after chemotherapy for metastatic testicular germ-cell tumors: a clinicopathological study of seven cases mandating separation from rhabdomyosarcoma
}

\author{
Jessica A Clevenger ${ }^{1}$, Richard S Foster ${ }^{2}$ and Thomas M Ulbright ${ }^{1 *}$ \\ ${ }^{1}$ Department of Pathology, Indiana University School of Medicine, Indianapolis, IN, USA and ${ }^{2}$ Department of \\ Urology, Indiana University School of Medicine, Indianapolis, IN, USA
}

\begin{abstract}
To gain insight concerning prognosis, we investigated seven cases of post-chemotherapy retroperitoneal lymph-node dissections from patients with testicular germ-cell tumors that contained sizable nodules of differentiated skeletal muscle, but that lacked both a primitive cellular component and mitotic activity. The patients were 18-28 years old at the time of retroperitoneal lymph-node dissection. All had a previous nonseminomatous germ-cell tumor of the testis, five of which had a teratoma component. In one the testicular tumor had foci of embryonal rhabdomyosarcoma. The retroperitoneal lymph-node dissections were performed 0.2-4.7 years after orchiectomy, all following cisplatin-based chemotherapy, and contained rhabdomyomatous tumors that ranged from $0.8-5 \mathrm{~cm}$. These consisted of nodular to diffuse aggregates of fetal-type rhabdomyocytes with central to peripheral nuclei and abundant, eosinophilic, fibrillary cytoplasm with occasional cross striations. Elongated myotubes with multiple nuclei in a common sarcoplasm occurred at least focally in all cases. Mild to moderate nuclear atypia, including nuclear enlargement and nucleolar prominence, was present, but mitotic activity, necrosis, and a primitive cellular component were absent. All but one retroperitoneal lymph-node dissection also contained other teratomatous elements. Follow-up in six patients showed three were disease free at 2.2-3.4 years; two developed recurrent teratoma at 1.3-3.7 years; and a sixth developed recurrent teratoma at 0.5 and 2 years, followed at 17 years by progressive tumor with elevated $\alpha$-fetoprotein. No patient with available follow-up developed progressive sarcoma. We conclude that rhabdomyomatous tumors in retroperitoneal lymph-node dissection specimens after chemotherapy for metastatic testicular germ-cell tumors show clinical behavior similar to teratoma rather than rhabdomyosarcoma. We believe the most likely explanation for the finding of pure rhabdomyomatous tumors in this setting, a phenomenon sometimes termed 'cytodifferentiation,' is selective persistence of differentiated tumor cells because of chemotherapy.

Modern Pathology (2009) 22, 1361-1366; doi:10.1038/modpathol.2009.108; published online 24 July 2009
\end{abstract}

Keywords: testicular germ-cell tumor; rhabdomyomatous tumor; rhabdomyosarcoma; teratoma; retroperitoneum

Sarcoma is an uncommon finding in post-chemotherapy retroperitoneal lymph-node dissection specimens and carries a poor prognosis, ${ }^{1-3}$ with

*Correspondence: Dr TM Ulbright, MD, Department of Pathology, Indiana University Medical Center, Clarian Pathology Laboratory, Room 4014, 350 W. 11th Street, Indianapolis, IN, 46202, USA.

E-mail: tulbrigh@iupui.edu

Received 14 April 2009; revised and accepted 26 May 2009; published online 24 July 2009 rhabdomyosarcoma being the most common form of sarcomatous transformation and usually behaving aggressively. ${ }^{2-4}$ Rarely, sizable nodules of differentiated skeletal muscle are found, and it is presently unclear if these rhabdomyomatous tumors behave as teratoma or sarcoma. In this study, we report our experience with seven cases of rhabdomyomatous tumors in post-chemotherapy retroperitoneal lymph-node dissection specimens from patients with metastatic testicular germ-cell tumors to gain 
insight into the prognostic significance of this finding.

\section{Materials and methods}

A case log search for retroperitoneal lymph-node dissection specimens having substantial foci of purely rhabdomyomatous elements and no nonteratomatous germ-cell tumor elements identified five cases that were confirmed on retrospective slide review. In addition, two cases were included that no longer had slides available for review, but that had originally been seen in consultation by the senior author, who utilized the same criteria for the recognition of rhabdomyomatous tumors as employed in this study. The gross descriptions and all available hematoxylin and eosin-stained slides of the retroperitoneal lymph-node dissection specimens were reviewed (7-43 slides per case). The numbers of sections of the rhabdomyomatous tumors that were available varied from 1-6 (mean, 3.2). The cases were evaluated for the largest rhabdomyomatous tumor dimension, the nature of any associated germ-cell tumor elements, the growth pattern, nuclear pleomorphism, multinucleation, nuclear position of the rhabdomyocytes, mitotic activity, necrosis, and the presence of a primitive cellular component. Cases of rhabdomyosarcoma, based on mitotic activity and/or the presence of a primitive cell component, were excluded. The distinction of rhabdomyomatous tumor from teratoma containing elements of skeletal muscle was the overgrowth of the rhabdomyocytic component, as a pure lesion, to the extent that the majority of a low power $(\times 4)$ field (Olympus BX40 microscope equipped with WH1 $\times 10$ oculars; field area $=23 \mathrm{~mm}^{2}$ ) was occupied, as has been suggested for the recognition of sarcomas in germ-cell tumors. ${ }^{4}$ Clinical data, including information regarding the testicular neoplasms, were obtained by review of pathology reports, referring physician correspondence and medical record reports. Additional follow-up in some cases was obtained by telephone conversation with the referring pathologist or clinician.

\section{Results}

The clinical and pathological features are summarized in Table 1. All of the patients had received cisplatin-based chemotherapy before retroperitoneal lymph-node dissection; none received previous radiation therapy. They ranged from 18-28 years at the time of retroperitoneal lymph-node dissection, and all were known to have had non-seminomatous germ-cell tumors of the testis from 0.2-4.7 years before retroperitoneal lymph-node dissection (Table 1). Five of the testicular primaries contained a component of teratoma, one did not, and this information was not available for one case. One of the testicular primaries contained a focus of embryonal rhabdomyosarcoma.

The mass containing the rhabdomyomatous tumors varied from $3.3-17.5 \mathrm{~cm}$ with the cut surfaces being solid and cystic, the former varying from tan to yellow to gray and having firm to soft consistencies. On microscopic examination, all of the retroperitoneal lymph-node dissection specimens except for one contained teratomatous elements in addition to the rhabdomyomatous tumors. These included the usual array of interwoven glandular structures, cartilaginous nodules, fibromuscular stroma, and occasional other tissue types. The rhabdomyomatous tumors measured $0.8-5 \mathrm{~cm}$ (the two largest estimated from the measured tumor size on the slides and the number of involved slides, see Table 1). They grew as large nodules (Figure 1) to dissecting aggregates (Figure 2) of differentiated rhabdomyocytes, with the former having circumscribed interfaces with the surrounding tissue

Table 1 Clinical and pathological features

\begin{tabular}{|c|c|c|c|c|c|}
\hline $\begin{array}{l}\text { Age at RPLND } \\
\text { (years) }\end{array}$ & $T G C T$ & $\begin{array}{c}\text { Interval to } R T \\
\text { (years) }\end{array}$ & $\begin{array}{l}\text { Size of RT } \\
\quad(\mathrm{cm})\end{array}$ & Rx after RPLND & $F / U$ after RPLND (years) \\
\hline 28 & $\mathrm{~S}, \mathrm{~T}$ & 0.3 & 2 & None & NED, 2.6 \\
\hline 23 & YST, CC, EC & 0.2 & 1.5 & None & NED, 3.4 \\
\hline 19 & $\mathrm{~T}$, scar & 0.5 & $2-3$ & None & NED, 2.2 \\
\hline 18 & YST, T, EC, CC & 0.7 & NA & None & RP T, 1.3; lung nodules ${ }^{\mathrm{a}}, 3.7$ \\
\hline 27 & T, YST & 4.7 & 5 & ChemoRx & Mediastinal T, 1.7 \\
\hline 27 & NSGCT & 0.5 & 0.8 & $\begin{array}{l}\text { ChemoRx at } 2 \text { years } 2 \text { nd } \\
\text { RPLND at } 2 \text { years Chemo Rx, } \\
16 \text { years }\end{array}$ & $\begin{array}{l}\text { Neck T, 0.5; elevated AFP, } 2 \text {; } \\
\text { RP T, 2; elevated AFP, 16; died of } \\
\text { tumor with elevated AFP, } 17.5\end{array}$ \\
\hline 18 & T, PNET, RMS, YST & 0.3 & NA & NA & Lost \\
\hline
\end{tabular}

AFP, $\alpha$-fetoprotein; CC, choriocarcinoma; EC, embryonal carcinoma; F/U, follow-up; NA, no information available; NED, no evidence of disease; NSGCT, non-seminomatous germ-cell tumor; PNET, primitive neuroectodermal tumor; RMS, rhabdomyosarcoma; RT, rhabdomyomatous tumor; $\mathrm{RP}$, retroperitoneal; RPLND, retroperitoneal lymph-node dissection; Rx, treatment; S, seminoma; T, teratoma; TGCT, testicular germ-cell tumor; YST, yolk sac tumor.

${ }^{\mathrm{a}}$ Clinically teratoma but not confirmed. 


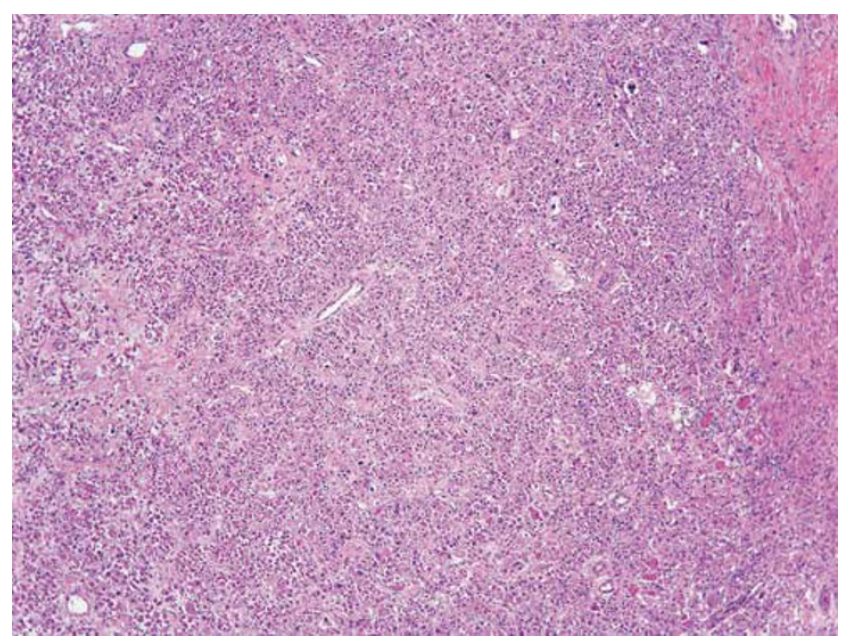

Figure 1 A large, circumscribed nodule of rhabdomyomatous tumor interfaces with adjacent smooth muscle (right).

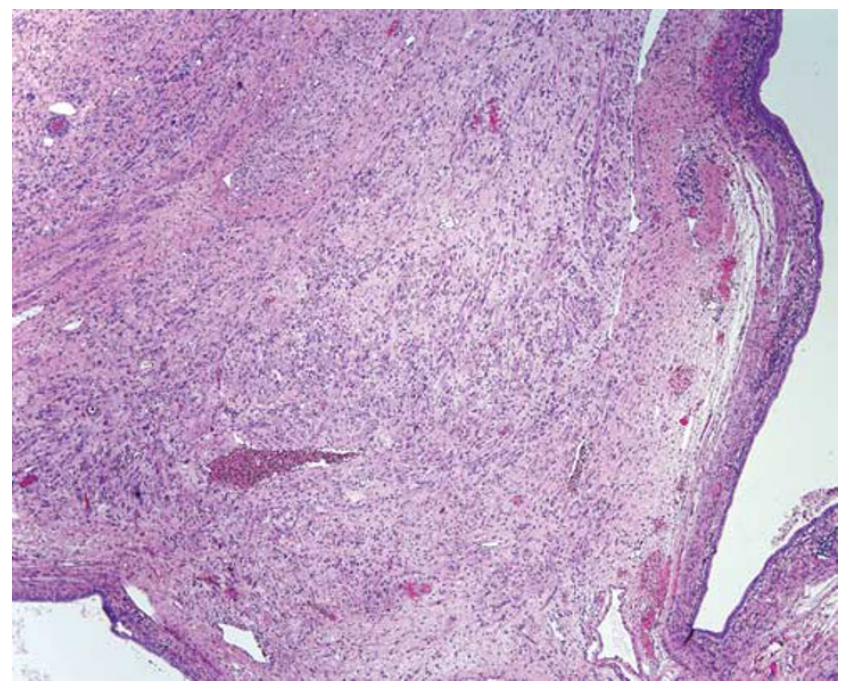

Figure 2 This rhabdomyomatous tumor dissects among teratomatous glands.

(Figure 3) and the latter showing peripheral infiltration between teratomatous elements (Figure 4). The cells had either round, regular profiles with centrally placed, spherical nuclei (Figure 5) or elongated, tubular profiles with peripherally placed nuclei (Figure 6). Such myotubes commonly showed nuclear aggregation in a common sarcoplasm (Figure 6) and were identified at least focally in every case. The nuclei showed mild to moderate pleomorphism and had small to moderate-sized nucleoli. Degenerate-type hyperchromasia occurred focally in two cases (Figure 7). Mitotic activity was not seen. In no case was there evident necrosis of the rhabdomyomatous elements. There was no evidence of a primitive, undifferentiated tumor component associated with the rhabdomyocytes.

Follow-up was available in six patients (Table 1). Four patients did not receive any additional

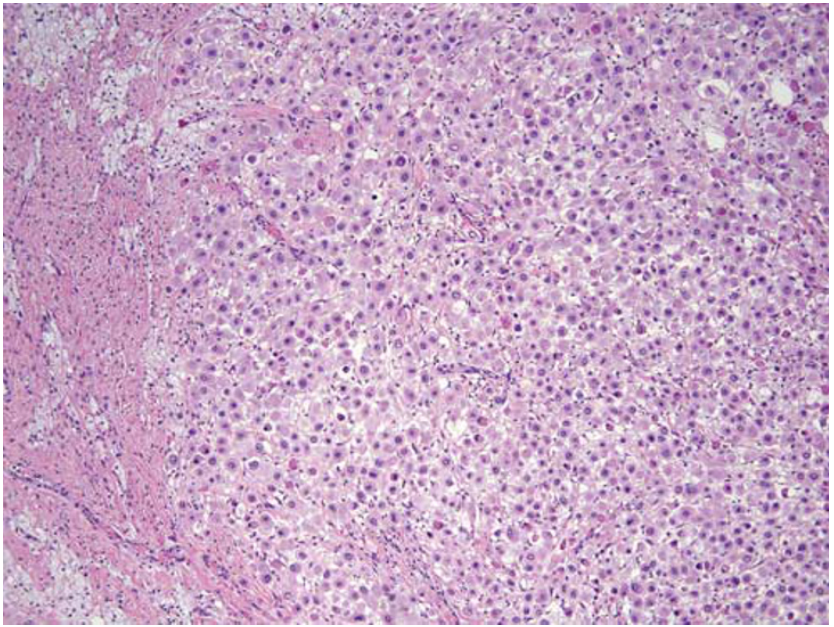

Figure 3 This nodule of rhabdomyomatous tumor has a circumscribed interface with adjacent fibrous tissue containing foamy histiocytes (left).

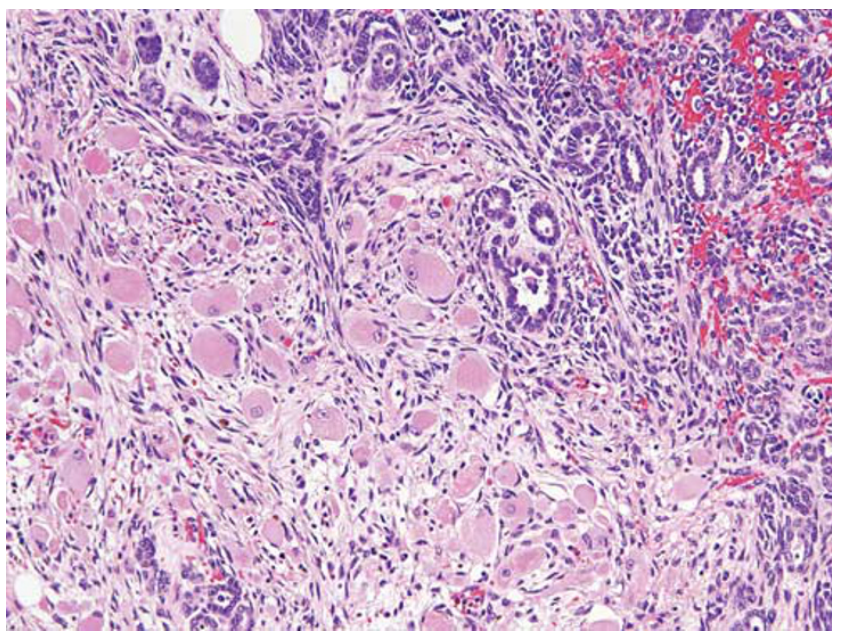

Figure 4 This rhabdomyomatous tumor infiltrates among embryonic-type renal elements.

treatment after retroperitoneal lymph-node dissection, and three had no evidence of disease from 2.2-3.4 years after retroperitoneal lymph-node dissection; the fourth recurred with retroperitoneal teratoma at 1.3 years after retroperitoneal lymphnode dissection and, 2.4 years later, with lung nodules that were clinically felt to represent teratoma, although this was not confirmed pathologically. A fifth patient, whose retroperitoneal lymph-node dissection had been interpreted as rhabdomyosarcoma, received additional chemotherapy after surgery and developed mediastinal teratoma at 1.7 years after retroperitoneal lymph-node dissection. The sixth patient did not receive any additional treatment until 2 years after retroperitoneal lymph-node dissection when serum AFP became elevated; he therefore received salvage chemotherapy followed by repeat retroperitoneal 


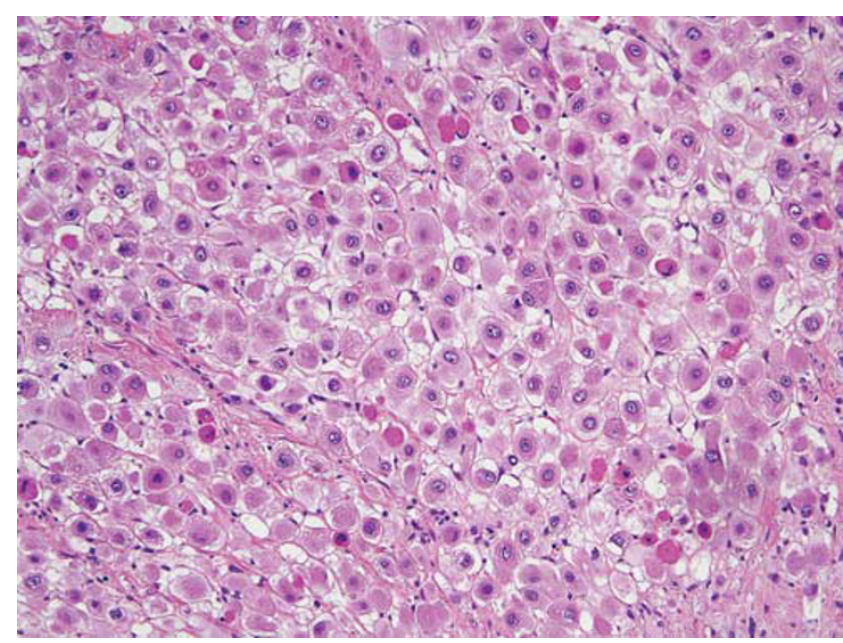

Figure 5 These round rhabdomyocytes have centrally placed, spherical nuclei and abundant eosinophilic cytoplasm.

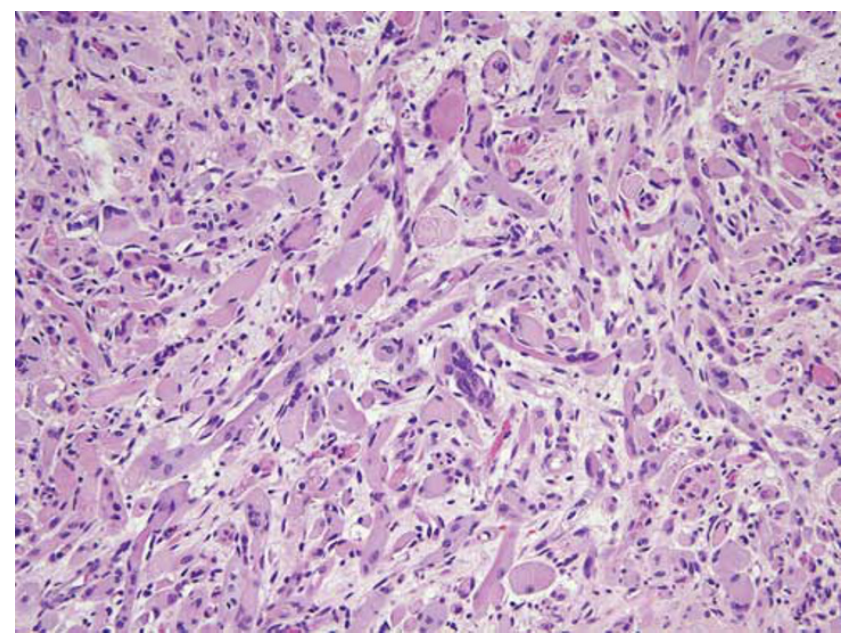

Figure 6 This tumor forms numerous myotubes with aggregated nuclei in a common sarcoplasm.

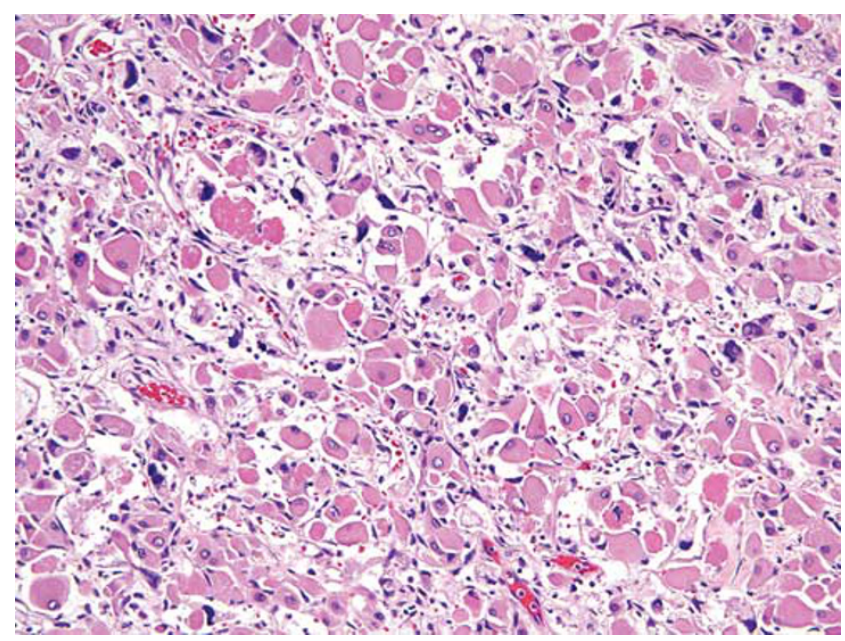

Figure 7 This tumor has numerous rhabdomyocytes with degenerate-type nuclear atypia. lymph-node dissection that showed only teratoma. He did well until 16 years after the initial retroperitoneal lymph-node dissection when serum AFP elevation again developed; additional chemotherapy was not effective and he died of tumor with progressively higher AFP levels at 17.5 years.

\section{Discussion}

Historically, teratoma in post-chemotherapy retroperitoneal lymph-node dissection specimens of patients with metastatic testicular germ-cell tumors is a good prognostic finding. Eggener et $a l^{5}$ show a 5 -year disease-specific survival of $87 \%$ in this circumstance. This figure is in line with the results of other studies that reported probabilities of remaining disease free of $83 \%$ at 5 years and $80 \%$ at 10 years, and 5 -year survival rates of $80-92 \% .{ }^{6,7}$

On the other hand, the presence of sarcoma in post-chemotherapy retroperitoneal lymph-node dissection specimens has a guarded prognosis. Little et $a l^{1}$ showed a disease-specific survival of $68 \%$ for various sarcomas at a mean follow-up of 2.5 years. Malagon et $a l^{2}$ reported only a $20 \%$ survival at a mean of 1.5 years for patients with germ-cell tumors having sarcomatous components in either the primary site or in their metastases. Comiter et $a l^{3}$ described an overall survival rate of $52 \%$ with a median follow-up of 4 years for teratomas with a secondary malignant component; however, those with rhabdomyosarcoma had a much poorer outcome, with only $25 \%$ achieving no evidence of disease status at a mean of 3.5 years.

Over the years we have seen rare examples of rhabdomyomatous tumors in post-chemotherapy retroperitoneal lymph-node dissections. When confronted with this finding it remained unclear whether such neoplasms should be considered equivalent to teratoma, sarcoma or an intermediate lesion regarding prognosis. For that reason, we have reviewed our experience with seven cases of rhabdomyomatous tumors that occurred in postchemotherapy retroperitoneal lymph-node dissections of patients with testicular germ-cell tumors. Although we made no attempt to determine the frequency of this finding in a systematic fashion, and this series is skewed by the selection bias that accompanies consultation cases, post-chemotherapy rhabdomyomatous tumors are rare, estimated at well under $1 \%$ of retroperitoneal lymph-node dissection specimens. The follow-up of our patients, although limited, showed $100 \%$ disease-specific survival (i.e., no patient deaths due to sarcoma). The only patient known to have died of tumor succumbed to progressive germ-cell tumor with marked AFP elevation at 17.5 years; presumably he had late recurrence of chemoresistant yolk sac tumor, although there was no histological documentation of this. From the prognostic viewpoint, therefore, this result is much more akin to 
the finding of teratoma in a post-chemotherapy retroperitoneal lymph-node dissection than rhabdomyosarcoma.

Similarly, lesions consisting solely of 'mature rhabdomyoblasts' (analogous to our rhabdomyomatous tumors) can occur in children who have been treated for rhabdomyosarcoma. This phenomenon of 'cytodifferentiation' in childhood rhabdomyosarcoma following chemotherapy has been recognized for 25 years. ${ }^{8}$ Molenaar et $a l^{8}$ reported a high proportion of differentiated tumor cells after chemotherapy in those childhood rhabdomyosarcomas that initially contained some such cells. Subsequent studies of cytodifferentiation in pediatric rhabdomyosarcomas reported its association with a good prognosis. Thus, Ortega et $a l^{9}$ identified six children who had residual, 'well-differentiated rhabdomyoblasts' after completion of therapy for pelvic rhabdomyosarcoma, and all were alive without evidence of disease at a follow-up period of 37-237 months. Arndt et al ${ }^{10}$ reported relapse in only 1 of 10 children with 'terminally differentiated rhabdomyoblastic lesions' after treatment for bladder/prostate rhabdomyosarcoma. And Smith et $a l^{11}$ reported no treatment failures in children with rhabdomyosarcomas whose tumors consisted of at least $70 \%$ differentiated cells after chemotherapy. This latter study suggests that a good prognosis in germ-cell tumor patients may not require a fully differentiated rhabdomyomatous tumor, but this is a question that our investigation cannot address because our exclusion criteria included any amount of primitive-appearing or mitotically active cells. However, the studies of the pediatric rhabdomyosarcomas and our results, collectively, indicate that highly differentiated rahbomyomatous tumors are a prognostically favorable finding.

The mechanism for the development of rhabdomyomatous tumors is not clear, but we feel it is most likely a chemoselection phenomenon wherein the mitotically active, primitive component of embryonal rhabdomyosarcoma is destroyed, with persistence of the differentiated tumor component. This mechanism has been previously suggested for the formation of teratomatous elements in post-chemotherapy specimens of patients with testicular germ-cell tumors, ${ }^{12}$ and is the pathogenesis suggested by Molenaar et $a l^{8}$ based on their study of childhood rhabdomyosarcomas. Others, however, postulate that chemotherapy itself may induce tumor differentiation. ${ }^{11,13}$ Based on the fact that most of the rhabdomyomatous tumors were a component of a lesion that also included more traditional teratoma and only one case had recognizable embryonal rhabdomyosarcoma in the testicular primary, we believe the rhabdomyomatous tumors usually developed from a metastatic germ-cell tumor that initially formed embryonal rhabdomyosarcoma (as well as other germ-cell tumor derivatives) in the retroperitoneum, with subsequent alteration of the morphology with chemotherapy.
Our results emphasize the importance of distinguishing post-chemotherapy rhabdomyomatous tumors from rhabdomyosarcoma. The key differential features, based on the criteria used in our study, are the absence of necrosis, mitotic activity, and a primitive cellular component in the former. These features are all characteristically seen in the rhabdomyosarcomas that occur in germ-cell tumor patients. Although several of our cases had relatively small amounts of the rhabdomyomatous tumor, there were two cases that measured $2-3 \mathrm{~cm}$ and $5 \mathrm{~cm}$, respectively. This implies, with admittedly limited data, that sizable amounts of rhabdomyomatous tumor do not necessarily have a more aggressive outcome. As skeletal muscle is a frequent element in post-chemotherapy teratomas, a distinction should be made between rhabdomyomatous tumor and teratoma with skeletal muscle elements. The minimal criterion, in our opinion, is overgrowth, with exclusion of other teratomatous elements, such that the majority of a low power $(\times 4)$ field is occupied by the rhabdomyocytic lesion. This is the criterion that is usually employed for the recognition of sarcomas that have developed in germ-cell tumors. ${ }^{2,4}$ All of the cases in this study had substantially more overgrowth than this minimal requisite amount.

In summary, our results support that pure rhabdomyocytic lesions in post-chemotherapy retroperitoneal lymph-node dissection specimens of patients with metastatic testicular germ-cell tumors are associated with a good prognosis when they are only associated with other teratomatous lesions. They should, therefore, not be diagnosed as 'welldifferentiated rhabdomyosarcoma'because this term in this context connotes a poor prognosis. The behavior, therefore, of such rhabdomyocytic neoplasms is similar to teratoma in the same context.

\section{Diclosure/conflict of interest}

The authors declare no conflict of interest.

\section{References}

1 Little Jr JS, Foster RS, Ulbright TM, Donohue JP. Unusual neoplasms detected in testicular cancer patients undergoing postchemotherapy retroperitoneal lymphadenectomy. World J Urol 1994;12:200-206.

2 Malagon HD, Valdez AM, Moran CA, Suster S. Germ cell tumors with sarcomatous components: a clinicopathologic and immunohistochemical study of 46 cases. Am J Surg Pathol 2007;31:1356-1362.

3 Comiter CV, Kibel AS, Richie JP, Nucci MR, Renshaw AA. Prognostic features of teratomas with malignant transformation: a clinicopathological study of 21 cases. J Urol 1998;159:859-863.

4 Ulbright TM, Loehrer PJ, Roth LM, Einhorn LH, Williams SD, Clark SA. The development of nongerm cell malignancies within germ cell tumors. 
A clinicopathologic study of eleven cases. Cancer 1984;54:1824-1833.

5 Eggener SE, Carver BS, Loeb S, Kondagunta GV, Bosl GJ, Sheinfeld J. Pathologic findings and clinical outcome of patients undergoing retroperitoneal lymph node dissection after multiple chemotherapy regimens for metastatic testicular germ cell tumors. Cancer 2007;109:528-535.

6 Dearnaley D, Huddart R, Horwich A. Regular review: managing testicular cancer. BMJ 2001;322:1583-1588.

7 Carver BS, Shayegan B, Serio A, Motzer RJ, Bosl GJ, Sheinfeld J. Long-term clinical outcome after postchemotherapy retroperitoneal lymph node dissection in men with residual teratoma. J Clin Oncol 2007;25: 1033-1037.

8 Molenaar WM, Oosterhuis JW, Kamps WA. Cytologic 'differentiation' in childhood rhabdomyosarcoma following polychemothcrapy. Hum Pathol 1983;15:973-979.

9 Ortega JA, Rowland J, Monforte H, Malogolowkin M, Triche T. Presence of well-differentiated rhabdomyoblasts at the end of therapy for pelvic rhabdomyosar- coma: Implications for the Outcome. J Pediatr Hematol Oncol 2000;22:106-111.

10 Arndt CA, Hammond S, Rodeberg D, Qualman S. Significance of persistent mature rhabdomyoblasts in bladder/prostate rhabdomyosarcoma: results from IRS IV. J Pediatr Hematol Oncol 2006;28:563-567.

11 Smith LM, Anderson JR, Coffin CM. Cytodifferentiation and clinical outcome after chemotherapy and radiation therapy for rhabdomyosarcoma (RMS). Med Pediatr Oncol 2002;38:398-404.

12 Oosterhuis JW, Suurmeyer AJH, Sleyfer DT, Koops HS, Oldhoff J, Fleuren G. Effects of multiple-drug chemotherapy (cis-diammine-dichloroplatinum, bleomycin and vinblastine) on the maturation of retroperitoneal lymph node metastases of nonseminomatous germ cell tumors of the testis: no evidence for de novo induction of differentiation. Cancer 1983;51: 408-416.

13 Nigro KG. Cytodifferentiation, but not resolution, of embryonal rhabdomyosarcoma. Pediatr Dev Pathol 2004;7:398-399. 